\title{
Ontology-based Concept Similarity Integrating Image Semantic and Visual Information
}

\author{
Mengyun Wang, Xianglong Liu, Lei Huang, Bo Lang, Hailiang Yu \\ State Key Laboratory of Software Development Environment \\ Beihang University, Beijing 100191, China \\ Email:\{jesuisenvie, xlong_liu, huanglei, langbo, yhl\}@nlsde.buaa.edu.cn
}

\begin{abstract}
In recent years, the concept similarity measure has received wide attention in many applications, such as ontology construction, text analysis, image retrieval, etc. Currently, the concept similarity measure depends on the information mining in various knowledge bases, like dictionaries, ontologies, image annotation labels, and search engines. However, these knowledge bases usually only contain semantic information. With the development of the Internet and the popularity of the digital imaging devices, a lot of images and related texts have appeared, which help us to further mine the concept similarity relationships. The concept similarity is the outcome of human subjective perception. In addition to analysis of semantic information, the content of image itself precisely provides the visual perception information, which also plays an important role in the access of concept similarity relationships. To integrate both image semantic and visual information, in this paper we propose an ontology concept similarity measure that simultaneously utilizes the image semantic annotations and visual features to optimize the ontology-based metrics. The experiment result on the Corel dataset demonstrates the effectiveness of our proposed method.
\end{abstract}

\section{INTRODUCTION}

$\mathrm{T}$ HE concept similarity plays a critical role in ontology construction and multimedia analysis. It is widely used in different researches and applications, such as ontology learning, semantic disambiguation, text clustering, and information annotation and retrieval.

Generally speaking, semantic similarity quantitatively describes the similarity degree between concepts. Traditional metrics assess the concept similarity relationships by exploiting one or several knowledge bases like corpus and ontologies. With the quick development of the Internet and the popularity of the digital imaging devices such as webcams, phone cameras, and digital cameras, a lot of images and related texts have emerged and formed a much rich knowledge base. For instance, Flickr provides free services for uploading and sharing images, where some necessary information such as titles, descriptions, and labels is usually required. As a large knowledge base, the text information can be used to evaluate the semantic similarity between labels [1]. In this paper, we consider the words 'label' and 'concept' refer to the same thing. However, such way only considers the correlation of text information around images, ignoring the indispensable effect of image visual information. Google search engine stores a wealth of network text resources, which can also help define the semantic similarity of concepts [2]. Compared with ontologies, there are several problems with concepts similarity measure only depending on the above knowledge bases: there is a lot of noisy data resulting inaccurate similarity measure; the concept definition is inexplicit, and cannot be used to distinguish synonyms or antonyms.

To address these problems, we propose an ontology concept similarity measure named OVS that simultaneously integrate both image semantic and visual information. Based on the ontology semantic similarity metrics, OVS exploits the images' visual features and related semantic annotations to optimize the semantic similarity relationships, which are more consistent with human cognition. Compared with traditional methods, the OVS integrates the semantic annotations around images with a variety of semantic knowledge in ontology such as hierarchical structure and semantic relationships, forming a richer semantic knowledge base. Meanwhile, we take images' visual information into consideration, utilizing visual knowledge base to optimize the concept similarity relationships. Integrating multiple knowledge bases together enables our approach to comprehensively express the semantic similarity relationships between concepts.

The main contributions of this paper are summarized as follows:

1. We propose a concept similarity measure by taking both images' visual features and semantic annotations into consideration.

2. We further propose an ontology-based concept similarity measure, which integrates both ontology semantic relationship and visual similarity measure.

The rest of this paper is organized as follows: Sec. II summarizes the existing ontology-based concept similarity measures. Sec. III introduces a novel ntology concept similarity measure named OVS based on the integration of image semantic and visual information. Sec. IV introduces the experiment and the last section is summarization of this thesis. 


\section{ONTOLOGY-BASED CONCEPT SIMILARITY MEASURES}

An ontology is a formal, explicit specification of a shared conceptualization [3]. 'Formal' refers to the fact that the ontology should be machine readable, while 'Shared' reflects the notion that an ontology captures consensual knowledge. The characteristics mentioned above make an ontology be a reliable structured knowledge base. With the rapid development of the Semantic Web, a large number of universal ontologies and domain ontologies are generated and widely applied to knowledge-based systems, particularly, the measure of concept similarity.

One of the most widely used ontologies is Wordnet. It is an English semantic dictionary which is domain-independent. A synset corresponds to a concepts. Wordnet describes more than 100,000 English concepts and multiple semantic relationships between those concepts, such as hyponymy, part-of, synonymy, and antonymy, of which the hyponymy occupies nearly $80 \%$. By connecting the related concepts together with multiple semantic relationships, Wordnet becomes a hierarchical structure or network structure. Based on this, researchers can mine the semantic similarity relationships of concepts with the methods like graph model.

Ontology-based concept similarity measures can be divided into four categories: the path-based method [4]-[7], the feature-based method [8], the IC(Information Content)-based method [9]-[12], and the gloss-based method [13][14]. In this section, we will introduce these methods and analyze the advantages and disadvantages of them.

Ontology can be modeled as a directed graph, in which a vertex represents a concept and an edge represents the hyponymy relation between two concepts. To calculate the similarity of concepts, the most direct method which is proposed by Rada et al. [4] is to compute the shortest path between two concepts, which follows the assumption that the concepts are more similar as the path is shorter. However, this method only considers the path between concepts, which cannot convey the similarity relationships of concepts precisely. Thus, several researches have taken the depth of the concept in the ontology into consideration [5][6]. Furthermore, multiple semantic relationships of concepts in the ontology are exploited, in addition to the hyponymy relations, to measure the semantic similarity relationships of concepts [7].

The advantage of the path-based method is the briefness in computation with the graph model, while faces the disadvantages that:

1. It only considers the shortest path between concepts within the ontology, ignoring the rich semantic knowledge of it.

2. The weight of every edge is identical in this approach. However, in the real sense, the semantic distance of each edge may not the same, which depends on the hierarchy granularity and degree of details described by concepts.
When two concepts are from different ontologies, the path-based method cannot calculate the semantic similarity of the concepts. Luckily, feature-based method can adapt to it. Feature-based method count the common part of property features between two concepts. If the common part is large, the concepts are similar, otherwise not. The property features can be extracted from a variety of semantic information like hypernym. This method describes the similarity degree of concepts more precisely by taking the common characteristic and the difference of concept properties into consideration. However, it usually depends on a large ontology like Wordnet.

In order to make up the lack of path-based measure, Resnik [9] proposed an IC-based method. He tried to utilize the IC shared by concepts to calculate the semantic similarity. IC can be derived from the frequency of concept appearance in a corpus, and the shared part of two concepts is represented by the LCS(Last Common Subsumer). But there is a problem: in terms of two concept-pairs with the same LCS, they will get the same similarity score. To address this problem, Lin [10] and Jiang \& Conrath [11] proposed methods to improve Resnik's method. They both considers the IC of two concepts as well as the IC of their LCS, to represent the concepts more comprehensively. The key point of this method is the access of IC, which depends on the corpus after text processing or the ontology with rich concepts. The accuracy of IC-based measure, to some extent, is influenced by the analysis of these knowledge bases.

The gloss-based method was first applied to semantic disambiguation. Lesk [15] compared the glosses of a word in a phrase with the glosses of others, finding the most similar sense as the sense of the word in this phrase. The glosses were described in a dictionary. Then Banerjee \& Pedersen [13] replaced it with Wordnet and introduced the ontology in. The premise of this method is the existence of a perfect corpus, including detailed gloss of words. Wordnet can satisfy the requirement which provides the hierarchical structure of concepts and abundant semantic glosses.

\section{ONTOLOGY CONCEPT SIMILARITY METHOD BASED ON IMAGE SEMANTIC ANNOTATIONS AND VISUAL FEATURES}

In Sec. II, we summarize different categories of the ontology-based concept similarity measures. In general terms, the measure of concept similarity is extracting a variety of semantic information based on different knowledge bases, to find the semantic similarity relationships between concepts. Ontology provides a variety of knowledge sources for semantic similarity measure, which contains a wealth of information on the concept gloss information and other semantic relations with a hierarchical structure based on the hyponymy relations. However, they are all semantic information based on text whether the glosses or the multiple semantic relations of concepts. As mentioned above, there are a lot of images and related semantic annotations on Flickr. Some researchers have tried to use the image semantic 
annotations to obtain the similarity relationship of labels. With the fast growing of images on the Internet and the research and application of ontology in image analysis, annotation and retrieval, the visual information of images is richer. And from a visual point of view, we are more likely to perceive whether two concepts are similar. Therefore, we propose an ontology concept similarity measure named OVS based on the integration of image semantic and visual information, which optimize the ontology-based method with images' semantic annotations and visual features, to obtain more consistent concept similarity relationships with human cognition.

\section{A. Semantic and Visual Relations based on Labeled Images}

The images in the web usually carry some labels expressing certain semantic concepts. Making use of annotation relationships between images and concepts in terms both semantic and visual correlations, we can obtain more powerful concept similarity measure.

Suppose there are $\mathrm{N}$ images, each of which is annotated by one or several labels from $\mathrm{M}$ concepts. Then we denote the pairwise similarity relationships between multiple concepts from both semantic and visual aspects using a symmetric matrix $R$ with each element $r_{i j}$ representing the similarity between concept $c_{i}$ and $c_{j}$. The similarity matrix $R$ consists of $a$ semantic relation matrix and a visual relation matrix respectively characterizing the correlations between concepts and images based on the semantic annotations and visual features.

The semantic relations matrix $\mathrm{S}$ is a matrix of $\mathrm{M} \times \mathrm{N}$. Each element of $\mathrm{S}$ is expressed as followed.

$\mathrm{s}_{\mathrm{ij}}=\left\{\begin{array}{cc}1 & \text { if image } \mathrm{j} \text { is annotated by concept } \mathrm{i} \\ 0 & \text { otherwise }\end{array}\right.$

Visual relations matrix $\mathrm{V}$ is also a matrix of $\mathrm{M} \times \mathrm{N}$. Each element of $\mathrm{V}$ represents the similarity between the visual features of concepts and images. Each concept $i$ corresponds to a subset of images annotated by it. The visual features of concept $i$ then can be described by the subset of images. We take the average of all these images' visual features from the subset as the visual feature of concept $i$. With the visual features of concept $i$ by $f_{i}$ and that of image $j$ represented by $f_{j}$, we calculate the element $v_{i j}$ of $\mathrm{V}$ as followed. In this paper, we choose SIFT to be the visual features of images, as it has demonstrated its out-performance in some benchmark evaluation comparing with various global features and local feature descriptors.

$$
v_{i j}=\cos \left(f_{i}, f_{j}\right)=\frac{\left\langle f_{i}, f_{j}\right\rangle}{\left\|f_{i}\right\|\left\|f_{j}\right\|}
$$

In practice, not every image shows strong similarity to its concept due to the visual variation of each concept, which will assign a lot of undesired images with small similarity to each concept in the dense matrix V. In order to make the visual relations matrix $\mathrm{V}$ robust to the variations, we only select the top k concepts with highest similarity for each image, thus forming a sparse visual relations matrix V. The smaller the value of $\mathrm{k}$, the more sparse the visual relations matrix $\mathrm{V}$ (the value of $\mathrm{k}$ is determined in Sec. IV.C.1).

\section{B. Concept Similarity based on Image Semantic and Visual Relations}

To incorporate both semantic and visual relations in the labeled image sets, we adopt a linear combination of $\mathrm{V}$ and $\mathrm{S}$ to get a comprehensive relations matrix VS,

$$
\mathrm{VS}=\mu * \mathrm{~V}+(1-\mu) * \mathrm{~S}
$$

where $\mu$ in $(0,1)$ is the weighting factor, which balances the contribution of the semantic and visual information (the value of $\mu$ is determined in Sec. IV.C.2) in the concept similarity measure.

Based on the fused relation matrix VS $=\left[\mathrm{y}_{1}, \ldots, \mathrm{y}_{\mathrm{m}}\right]^{\mathrm{T}}$, where the $\mathrm{m}$-th row $\mathrm{y}_{\mathrm{m}}$ is the comprehensive relations vector of concept $\mathrm{m}$. If two concepts share close semantics, then their comprehensive relations vectors should be very similar. Therefore, we employ the cosine similarity between the comprehensive relation vectors as the semantic similarity between two concepts.

$$
r_{i j}^{v s}=\cos \left(y_{i}, y_{j}\right)=\frac{\left\langle y_{i}, y_{j}\right\rangle}{\left\|y_{i}\right\|\left\|y_{j}\right\|}
$$

By calculating the pairwise similarities between all concepts, we can obtain the similarity matrix $\mathrm{R}$ based on image semantic annotations and visual features. The above method for the concept similarity measure is named VS method.

In practice, the semantic annotations of the image are often incomplete, which leads to the sparsity of the semantic relations matrix $\mathrm{S}$. In this case, the method only relying on image semantic annotations hardly obtains an accurate semantic similarity relationships of concepts. However, the VS method introduces the image visual information in Sec. III.A which hopefully compensates for the lack of semantic information.

One problem in the process of calculating the visual relations matrix $\mathrm{V}$ when only a few limited labels are available is that, the incompleteness of the semantic information will result in the bias of the visual feature center of each concept, which will adversely affect the visual relations matrix V. To solve the problem, attempts(like EM algorithms) that iteratively find the near-optimal visual feature center of each concept can be adopted.

\section{Ontology Metric with the Concept Similarity}

We have presented a concept similarity measure VS based on image semantic annotations and visual features. In this section, we will utilize it to optimize the metrics based on ontology. Ontology provides a wealth of semantic knowledge, especially the universal ontology (e.g. Wordnet), which does 
not depend on specific areas and is more consistent with the human conception. On the other hand, the human visual perception is also the important factor that affects the judgment of the concept similarity. The visual feature of the image is one general way to precisely express the concept of human visual perception. Therefore, fusing the combination of both semantic and visual relations into the traditional ontology metric can effectively compensate for the lack of the rich and complex relations in ontology.

Specifically, we adopt the parameter weighting method [16]-[18] to calibrate the ontology similarity metric using the concept similarity $\mathrm{R}$ based on the semantic relations and visual relations extracted from a number of labeled images. Since the sparse semantic annotations in images usually introduce certain bias in the similarity measure, it is better to fuse the ontology metric and concept similarity measure in a robust way. In this paper, we propose a novel method named OVS in short, which adopt the popular exponential production to robustly incorporate the visual-semantic relations in the ontology metric. Namely, for any two concepts $c_{i}$ and $c_{j}$, their final ontology-based concept similarity is as followed.

$$
r_{i j}^{\text {OVS }}=r_{i j}^{O} \exp \left(r_{i j}^{V S}\right)
$$

In the above formula, $r_{o}$ is the concept similarity based on ontology while $r_{v s}$ is the concept similarity based on image semantic and visual information.

\section{EXPERIMENT}

\section{A. Methods for Comparison}

In order to verify the effectiveness of the method OVS we propose, we conduct several comparative experiments with two kinds of metrics, which are metrics based on the ontology and metrics based on the image semantic and visual information, as shown in Table I.

TABLE I.

METHODS FOR COMPARISON

\begin{tabular}{|c|c|c|c|c|}
\hline Knowledge base & Method & Type & Published in & Formula \\
\hline \multirow{14}{*}{ Ontology } & WUP [5] & Path & 1994 & $\operatorname{sim}_{\mathrm{wUP}}=\frac{2 * \mathrm{~N}_{3}}{\mathrm{~N}_{1}+\mathrm{N}_{2}+2 * \mathrm{~N}_{3}}$ \\
\hline & LCH [6] & Path & 1998 & $\operatorname{sim}_{\mathrm{LCH}}=-\log \left(\frac{\mathrm{N}}{2 * \mathrm{D}}\right)$ \\
\hline & HSO [7] & Path & 1998 & $\operatorname{sim}_{\mathrm{HSO}}=\mathrm{C}-$ full $\_$path $\left(\mathrm{c}_{1}, \mathrm{c}_{2}\right)-\mathrm{k} * \operatorname{turns}\left(\mathrm{c}_{1}, \mathrm{c}_{2}\right)$ \\
\hline & RAD [4] & Path & 1989 & $\operatorname{dis}_{\mathrm{RAD}}=\min _{\forall \mathrm{i}}\left|\mathrm{p}_{\mathrm{i}}\left(\mathrm{c}_{1}, \mathrm{c}_{2}\right)\right|$ \\
\hline & SAN [8] & Feature & 2012 & $\begin{array}{l}\operatorname{dis}_{\mathrm{SAN}}=\log _{2}\left(1+\left(\left|\phi\left(\mathrm{c}_{1}\right) \backslash \phi\left(\mathrm{c}_{2}\right)\right|+\left|\phi\left(\mathrm{c}_{2}\right) \backslash \phi\left(\mathrm{c}_{1}\right)\right|\right)\right. \\
\left./\left(\left|\phi\left(\mathrm{c}_{1}\right) \backslash \phi\left(\mathrm{c}_{2}\right)\right|+\left|\phi\left(\mathrm{c}_{2}\right) \backslash \phi\left(\mathrm{c}_{1}\right)\right|+\left|\phi\left(\mathrm{c}_{1}\right) \cap \phi\left(\mathrm{c}_{2}\right)\right|\right)\right)\end{array}$ \\
\hline & $\mathrm{JCN}[11]$ & IC & 1997 & $\operatorname{dis}_{\mathrm{JCN}}\left(\mathrm{c}_{1}, \mathrm{c}_{2}\right)=\left(\mathrm{IC}\left(\mathrm{c}_{1}\right)+\mathrm{IC}\left(\mathrm{c}_{2}\right)\right)-2 * \operatorname{sim}_{\mathrm{RES}}\left(\mathrm{c}_{1}, \mathrm{c}_{2}\right)$ \\
\hline & RES [9] & IC & 1995 & $\operatorname{sim}_{\mathrm{RES}}\left(\mathrm{c}_{1}, \mathrm{c}_{2}\right)=\mathrm{IC}\left(\operatorname{LCS}\left(\mathrm{c}_{1}, \mathrm{c}_{2}\right)\right)$ \\
\hline & LIN[10] & IC & 1998 & $\operatorname{sim}_{\mathrm{LIN}}\left(\mathrm{c}_{1}, \mathrm{c}_{2}\right)=\frac{2 * \operatorname{sim}_{\mathrm{RES}}\left(\mathrm{c}_{1}, \mathrm{c}_{2}\right)}{\mathrm{IC}\left(\mathrm{c}_{1}\right)+\operatorname{IC}\left(\mathrm{c}_{2}\right)}$ \\
\hline & JCN_SAN [12] & IC & \multirow{3}{*}{2013} & \multirow{3}{*}{$\begin{array}{l}\mathrm{IC}_{\mathrm{SAN}}(\mathrm{c})=-\log \mathrm{p}(\mathrm{c}) \cong \\
-\log \left(\frac{\frac{|\operatorname{leaves}(\mathrm{c})|}{|\operatorname{subsumers}(\mathrm{c})|}+1}{\max \_ \text {leaves }+1}\right)\end{array}$} \\
\hline & RES_SAN [12] & IC & & \\
\hline & LIN_SAN [12] & IC & & \\
\hline & LESK [13] & Gloss & 2002 & - \\
\hline & VECTOR [14] & Gloss & 2006 & - \\
\hline & VECTOR_PAIRS [14] & Gloss & 2006 & - \\
\hline Image & VS & - & Sec. III.B & - \\
\hline
\end{tabular}




\section{B. Evaluation Benchmark and Indicator}

The objective evaluation of concept similarity is very difficult as the concept similarity is a human's subjective perception. To compare the methods fairly, some scholars have constructed artificial evaluation datasets as the groundtruth. This dataset contains a number of concept-pair, judge by a group of people with a similarity score. The average of valid data is the similarity of the concept-pair. In early, Rubenstein \& Goodenough [19] and Miller \& Charles [20] constructed those datasets, which are widely used to evaluate and compare the similarity measure. The current popular datasets are WordSim-353 [21], MEN [22], RWS [23], etc. MEN is constructed by Elia Bruni, containing 3000 concept-pairs with high frequency of appearance, in which the similarity is in the range of $0-50$. We choose Corel [24] to calculate the concept similarity, which contains 260 concepts. Based on the consideration of the above two aspects, we selected overlap portions of MEN and Corel as the target concept, which contains a total of 96 concepts, composing 118 concept-pairs.

Considering the artificial evaluation of concept similarity and the metric result as two sequences, their relevance is the indicator to judge semantic similarity metrics. The correlation is 1 if the scores are both exactly the same, which means that the results of the semantic metrics is consistent with human perception, while correlation of 0 means that the result of semantic metrics and the result of human perception are completely irrelevant. Currently the most widely used methods are the Pearson and Spearman correlation coefficient. Pearson correlation coefficient is calculated as

$$
\rho_{\mathrm{p}}=\frac{\mathrm{N} \sum \mathrm{x}_{\mathrm{i}} \mathrm{y}_{\mathrm{i}}-\sum \mathrm{x}_{\mathrm{i}} \sum \mathrm{y}_{\mathrm{i}}}{\sqrt{\mathrm{N} \sum \mathrm{x}_{\mathrm{i}}^{2}-\left(\sum \mathrm{x}_{\mathrm{i}}\right)^{2}} \sqrt{\mathrm{N} \sum \mathrm{y}_{\mathrm{i}}^{2}-\left(\sum \mathrm{y}_{\mathrm{i}}\right)^{2}}}
$$

In the above formula, $\mathrm{x}_{1}, \mathrm{x}_{2}, \ldots, \mathrm{x}_{\mathrm{n}}$ and $\mathrm{y}_{1}, \mathrm{y}_{2}, \ldots, \mathrm{y}_{\mathrm{n}}$ are the result of human judgment and semantic metric. Spearman correlation coefficient is usually considered to be the Pearson correlation coefficient between the variables after ranking. We need to rank the sequence first. If the rank is identical, the Pearson and Spearman correlation coefficients are equivalent according to the equation (6). Otherwise it is calculated according to the following formula,

$$
\rho_{\mathrm{s}}=1-\frac{6 \sum_{\mathrm{i}=1}^{\mathrm{N}} \mathrm{d}_{\mathrm{i}}^{2}}{\mathrm{~N}\left(\mathrm{~N}^{2}-1\right)}
$$

where $d_{i}$ is the difference of $x_{i}$ and $y_{i}$.

\section{Results and Discussion}

In order to verify the effectiveness of the method OVS, we conduct several comparative experiments with two kinds of metrics, which are metrics based on the ontology and metrics based on the image semantic and visual information.
1) The Impact of the Sparse Degree of Visual Relations Matrix

In Sec. III.A, we consider removing the interference data of visual relations matrix $\mathrm{V}$, and only selecting the first $\mathrm{k}$ concepts with highest similarity of the image, thus forming a sparse visual relations matrix V. The smaller the value of $\mathrm{k}$ is, the higher the sparse degree of $\mathrm{V}$ is. In this section, we take the experiment to evaluate the value of $\mathrm{k}$.

Parameter $\mu$ is the weight factor for the image semantic and visual information. $\mu=0$ means to calculate the semantic similarity matrix $\mathrm{R}$ with only image semantic annotations, while $\mu=1$ means to calculate $\mathrm{R}$ with only image visual features. We take the case of $\mu=0$ as our basis, to calculate the top 10 similar concepts of each concept on Corel. And then we calculate the top 10 similar concepts with different values of $\mathrm{k}$ in the case of $\mu=1$. After all, the accuracy of the top 10 similar concepts with different values of $k$, with respect to the case of $\mu=0$ is seen as the index to judge the sparse degree of $\mathrm{V}$. As the number of labels belonged to a image is limited, we set the range of $\mathrm{k}$ as 10 or less. The result is shown in Fig. 1.

As Fig. 1 shows, with the increasing of $\mathrm{k}$, the sparse degree of $\mathrm{V}$ decreases and the accuracy of top 10 similar concepts decreases accordingly. It is obvious that the value of $\mathrm{k}$ should be as small as possible. But if the value of $\mathrm{k}$ is too small, it will cause the over lost of the information in V. So in the following experiment, we take $\mathrm{k}=5$.

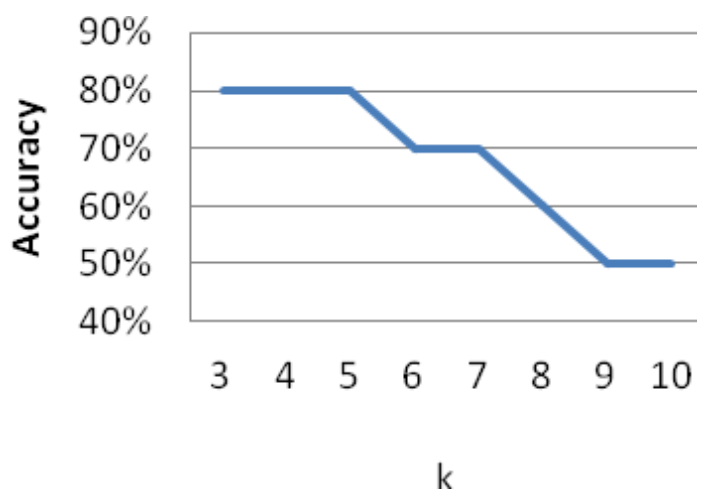

Fig. 1 The accuracy of top 10 similar concepts with different values of $\mathrm{k}$ 


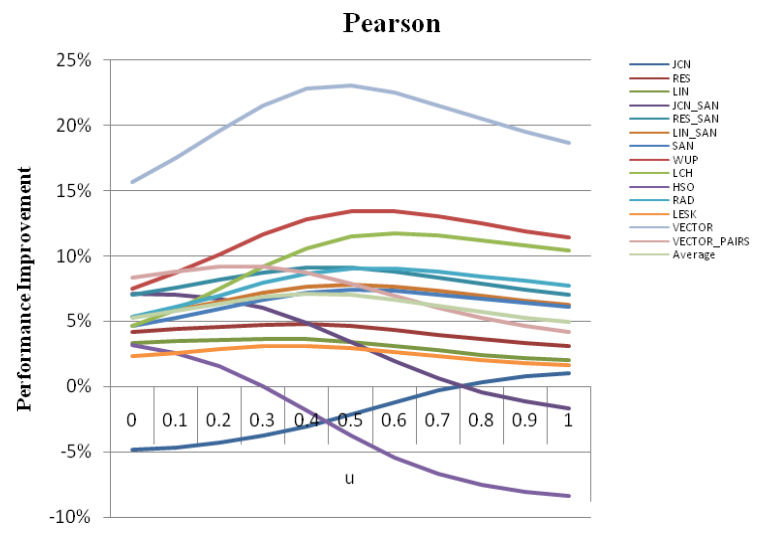

Fig. 2 Performance Improvement of Pearson correlation coefficients

\section{2) The Choice of Weighting Factor $\mu$}

In Sec. IV.B, we propose the evaluation benchmark and index of semantic similarity metrics. First, we calculate the semantic similarities of the target concept-pairs with the ontology-based metrics listed in Table I, and then calculate the correlation coefficients of every single result of those metrics with human judgment. Secondly, based on those

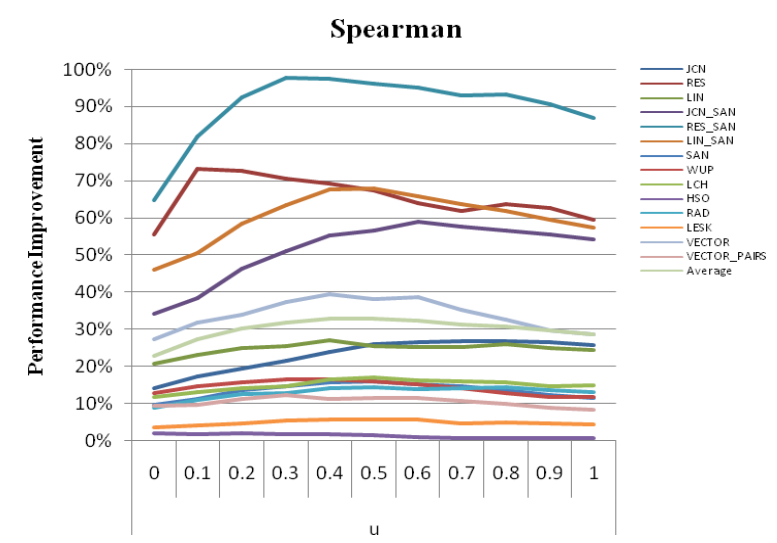

Fig. 3 Performance Improvement of Spearman correlation coefficients

ontology metrics in Table 1, we utilize OVS method proposed in Sec. III.C we calculate the semantic similarities of the target concept-pairs separately, and then calculate the correlation coefficients of these results with human judgment. Finally, we calculate the correlation coefficients promotion of results based on OVS with respect to the results of ontology-based metrics, and the result is shown in Fig. 2 and Fig. 3.

As Fig. 2 and Fig. 3 shows, each curve represents the change trend of the correlation coefficients promotion of the

TABLE II.

COMPARISON OF OVS WITH METHODS BASED ON ONTOLOGY AND IMAGE SEMANTIC AND VISUAL INFORMATION OVS

\begin{tabular}{|c|c|c|c|c|c|}
\hline \multirow{2}{*}{ Method } & \multirow{2}{*}{ Type } & \multicolumn{2}{|c|}{ Pearson } & \multicolumn{2}{|c|}{ Spearman } \\
\hline & & Ontology & OVs & Ontology & OVs \\
\hline WUP & Path & 0.3847 & 0.4363 & 0.3678 & 0.4271 \\
\hline $\mathrm{LCH}$ & Path & 0.4778 & 0.5327 & 0.4600 & 0.5382 \\
\hline HSO & Path & 0.4353 & 0.4189 & 0.4759 & 0.4833 \\
\hline RAD & Path & 0.4093 & 0.4461 & 0.4600 & 0.5269 \\
\hline SAN & Feature & 0.3800 & 0.4081 & 0.3662 & 0.4244 \\
\hline JCN & IC & 0.1254 & 0.1227 & 0.3329 & 0.4200 \\
\hline RES & IC & 0.3122 & 0.3266 & 0.1454 & 0.2435 \\
\hline LIN & IC & 0.3216 & 0.3325 & 0.2080 & 0.2613 \\
\hline JCN_SAN & IC & 0.2164 & 0.2239 & 0.2639 & 0.4134 \\
\hline RES_SAN & IC & 0.3081 & 0.3361 & 0.1495 & 0.2933 \\
\hline LIN_SAN & IC & 0.3262 & 0.3518 & 0.1718 & 0.2883 \\
\hline LESK & Gloss & 0.3711 & 0.3821 & 0.5562 & 0.5879 \\
\hline VECTOR & Gloss & 0.3387 & 0.4167 & 0.2536 & 0.3507 \\
\hline VECTOR_PAIRS & Gloss & 0.3514 & 0.3792 & 0.3624 & 0.4045 \\
\hline VS & Image & - & 0.2919 & - & 0.3533 \\
\hline
\end{tabular}


OVS method based on every ontology-based metrics with different value of $\mu$. Due to the different methods to get the concept similarity, which leads to different rank, Pearson and Spearman correlation coefficients are not the same. But the changing trend of promotion with different value of $\mu$ is almost the same. In Fig. 2, except that the Pearson promotions of OVS based on JCN, HSO, JCN_SAN metrics vary monotonically with different value of $\mu$, the promotions of OVS based on other ontology-based metrics get the maximum value with the value of $\mu \in(0.3,0.6)$. We also can find the similar conclusion for Spearman promotion. Based on the analysis above, in the sequent experiments, we take the optimal value $\mu=0.5$.

\section{3) Evaluation of Concept Similarity Method}

As Fig. 2 and Fig. 3 shows in Sec. IV.C.2, the concept similarities based on OVS get a promotion with respect to ontology-based metrics, except JCN, HSO and JCN_SAN. We also can get the same conclusion from Table II.

In addition, we can also get the idea from Table II that the path-based and gloss-based methods among the ontology-based methods have the best effect whether they are based on ontologies alone or based on the OVS, especially for the LCH metric, while IC-based methods have the worst. However, the IC-based methods get a promotion after the optimization of OVS. It is clear that the OVS method integrating the image semantic annotation and visual features together can achieve better performance than ontology-based methods. We also can find that in most cases the OVS method based on ontology metrics have a better effect than the VS method we proposed in Sec. III.A. Thus, we believe that the concept similarity relationships derived from ontologies and image semantic and visual information together is superior to the methods of ontologies or image semantic and visual information.

\section{V.CONCLUSION}

In this paper, we summarize the concept similarity methods based on ontology, and presents an ontology-based concept similarity method OVS integrating the image semantic and visual information. Firstly, OVS get concept similarities with different ontology-based metrics, then compute another concept similarity with the image semantic annotations and visual features as a knowledge base, and finally integrate the two kinds of measurement, constituting a new concept similarity measure. In the experiments, we discuss and determine the OVS method parameter value and compare the methods based on ontology and image semantic and visual information with the evaluation benchmark of human judgment. The result verifies the effectiveness of the proposed method OVS.

In this paper, we measure the concept similarity relationships on the Corel dataset, which contains less visual information than the images in the network. As future work, we plan to apply our method to large datasets like Imagenet and applications like image annotation and retrieval.

\section{REFERENCES}

[1] Xu H, Zhou X, Wang M, et al. Exploring Flickr's related tags for semantic annotation of web images. Proceedings of the ACM International Conference on Image and Video Retrieval. ACM, 2009: 46. DOI: http://dx.doi.org/10.1145/1646396.1646450

[2] Cilibrasi R L, Vitanyi P M B. The google similarity distance. Knowledge and Data Engineering, IEEE Transactions on, 2007, 19(3): 370-383. DOI: http://dx.doi.org/10.1109/TKDE.2007.48

[3] Studer R, Benjamins V R, Fensel D. Knowledge engineering: principles and methods. Data \& knowledge engineering, 1998, 25(1): 161-197. DOI: http://dx.doi.org/10.1016/S0169-023X(97)00056-6

[4] Rada R, Mili H, Bicknell E, et al. Development and application of a metric on semantic nets. Systems, Man and Cybernetics, IEEE Transactions on, 1989, 19(1): 17-30. DOI: http://dx.doi.org/10.1109/21.24528

[5] Wu Z, Palmer M. Verbs semantics and lexical selection. Proceedings of the 32nd annual meeting on Association for Computational Linguistics. Association for Computational Linguistics, 1994: 133-138. DOI: http://dx.doi.org/10.3115/981732.981751

[6] Leacock C, Chodorow M. Combining local context and WordNet similarity for word sense identification. WordNet: An electronic lexical database, 1998, 49(2): 265-283.

[7] Hirst G, St-Onge D. Lexical chains as representations of context for the detection and correction of malapropisms. WordNet: An electronic lexical database, 1998, 305: 305-332.

[8] Sánchez D, Batet M, Isern D, et al. Ontology-based semantic similarity: A new feature-based approach. Expert Systems with Applications, 2012, 39(9): 7718-7728. DOI: http://dx.doi.org/10.1016/j.eswa.2012.01.082

[9] Resnik P. Using information content to evaluate semantic similarity in a taxonomy. arXiv preprint cmp-lg/9511007, 1995.

[10] Lin D. An information-theoretic definition of similarity. ICML. 1998, 98: 296-304.

[11] Jiang J J, Conrath D W. Semantic similarity based on corpus statistics and lexical taxonomy. arXiv preprint cmp-lg/9709008, 1997.

[12] Sánchez D, Batet M. A semantic similarity method based on information content exploiting multiple ontologies. Expert Systems with Applications, 2013, 40(4): 1393-1399. DOI: http://dx.doi.org/10.1016/j.eswa.2012.08.049

[13] Banerjee S, Pedersen T. An adapted Lesk algorithm for word sense disambiguation using WordNet[M]. Computational linguistics and intelligent text processing. Springer Berlin Heidelberg, 2002: 136-145. DOI: http://dx.doi.org/10.1007/3-540-45715-1_11

[14] Patwardhan S, Pedersen T. Using WordNet-based context vectors to estimate the semantic relatedness of concepts. Proceedings of the EACL 2006 Workshop Making Sense of Sense-Bringing Computational Linguistics and Psycholinguistics Together. 2006, 1501: 1-8.

[15] Lesk M. Automatic sense disambiguation using machine readable dictionaries: how to tell a pine cone from an ice cream cone. Proceedings of the 5th annual international conference on Systems documentation. ACM, 1986: 24-26. DOI: http://dx.doi.org/10.1145/318723.318728

[16] Tversky A. Features of similarity. Psychological review, 1977, 84(4): 327. DOI: http://dx.doi.org/10.1037//0033-295X.84.4.327

[17] Rodríguez M A, Egenhofer M J. Determining semantic similarity among entity classes from different ontologies. Knowledge and Data Engineering, IEEE Transactions on, 2003, 15(2): 442-456.

[18] Zhou Z, Wang Y, Gu J. A new model of information content for semantic similarity in WordNet. Future Generation Communication and Networking Symposia, 2008. FGCNS'08. Second International Conference on. IEEE, 2008, 3: 85-89. DOI: http://dx.doi.org/10.1109/FGCNS.2008.16

[19] Rubenstein H, Goodenough J B. Contextual correlates of synonymy. Communications of the ACM, 1965, 8(10): 627-633. DOI: http://dx.doi.org/10.1145/365628.365657 
[20] Miller G A, Charles W G. Contextual correlates of semantic similarity. Language and cognitive processes, 1991, 6(1): 1-28. DOI: http://dx.doi.org/10.1080/01690969108406936

[21] Finkelstein L, Gabrilovich E, Matias Y, et al. Placing search in context: The concept revisited. Proceedings of the 10th international conference on World Wide Web. ACM, 2001: 406-414. DOI: http://dx.doi.org/10.1145/371920.372094

[22] Bruni E, Boleda G, Baroni M, et al. Distributional semantics in Technicolor. Proceedings of the 50th Annual Meeting of the
Association for Computational Linguistics: Long Papers-Volume 1. Association for Computational Linguistics, 2012: 136-145.

[23] Luong M T, Socher R, Manning C D. Better word representations with recursive neural networks for morphology. CoNLL-2013, 2013, 104.

[24] Bache, K. \& Lichman, M. (2013). UCI Machine Learning Repository [http://archive.ics.uci.edu/ml]. Irvine, CA: University of California, School of Information and Computer Science. 